\title{
Fotocoagulação a laser de diodo para tratamento de alteração vascular em lábio: relato de caso
}

Photocoagulation of diode laser for treatment of vascular lesion in lip: case report

Fotocoagulación a laser de diodo para tratamiento de alteración vascular en lábio: relato de caso

Fernanda Suely Barros DANTAS ${ }^{1}$

Letícia Targino CAMPOS $^{2}$

Raissa Lima TOSCANO ${ }^{2}$

Gabriella de Vasconcelos NEVES ${ }^{3}$

Gustavo Gomes AGRIPINO ${ }^{4}$

Daliana Queiroga de Castro GOMES ${ }^{4}$

${ }^{I}$ Mestranda pelo Programa de Pós-Graduação em Odontologia (PPgO), Departamento de Odontologia, Universidade Estadual da Paraíba (UEPB) 58429-500 Campina Grande- PB, Brasil

${ }^{2}$ Cirurgiã-Dentista, Departamento de Odontologia, Universidade Estadual da Paraíba (UEPB) 58429-500 Campina Grande- PB, Brasil

${ }^{3}$ Doutoranda pelo Programa de Pós-Graduação em Odontologia (PPgO), Departamento de Odontologia, Universidade Estadual da Paraíba (UEPB) 58429-500 Campina Grande- PB, Brasil

${ }^{4}$ Professor(a) Doutor(a) em Área de Estomatologia, Departamento de Odontologia, Universidade Estadual da Paraíba (UEPB) 58429-500 Campina Grande- PB, Brasil

\section{Resumo}

Introdução: As alterações vasculares são anomalias de desenvolvimento dos vasos que proporcionam acúmulo de sangue e são de etiologia desconhecida. Fazem parte deste grupo de lesões as malformações arteriovenosas, hemangiomas, linfoangiomas e varicosidades. Embora não raras, as lesões vasculares que ocorrem nos lábios, por vezes, interferem na estética e apresentam dificuldades quanto ao seu diagnóstico e tratamento. Objetivo: Descrever um caso clínico realizado com laser de diodo de alta potência para a fotocoagulação de uma lesão vascular, abordando suas vantagens e limitações em comparação ao tratamento cirúrgico. Material e Método: O procedimento foi realizado em um paciente de 53 anos, sexo feminino, branca, com queixa de aparecimento de uma bolha de sangue no lábio com evolução de aproximadamente oito anos. Ao exame físico intraoral, observou-se nódulo em vermelhão do lábio inferior, do lado direito, próxima à comissura labial, medindo aproximadamente $1,0 \mathrm{~cm}$ de diâmetro, de base séssil, superfície lisa, limites bem definidos, coloração violácea, consistência macia e sem sintomatologia dolorosa à palpação. Após a diascopia e diagnóstico clínico de lesão vascular, optou-se pela fotocoalulação com laser de diodo de alta potência. Resultados: Houve regressão total da lesão, após uma única aplicação, sem complicações trans e pós-operatórias, sem sangramento durante a cirurgia, o que proporcionou melhor visão do campo operatório e resultou em um procedimento minimamente invasivo. A paciente encontra-se em proservação, sem recidiva da lesão. Conclusão: A fotocoagulação pode ser considerada como uma alternativa segura e eficiente para o tratamento de lesões vasculares.

Descritores: Lesões do Sistema Vascular; Fotocoagulação; Laser; Mucosa Bucal.

\section{Abstract}

Introduction Vascular alterations are developmental anomalies of vessels resulting in blood accumulation, and whose etiology is unknown. This group of lesions includes Arteriovenous malformations, Hemangiomas, Lymphoangiomas, and Varicosities. Though not rare, labial vascular lesions sometimes interfere with aesthetic and present difficulties as for their diagnosis and treatment. Objective: The main goal of this study is to describe a clinical case performed by using high-power diode laser for the photocoagulation of a vascular lesion, highlighting its advantages and limitations as compared to those of the surgical treatment. Material and Method: The procedure was performed in a 53year-old, female, white patient who had complaints of a blood bubble on the lip with an eight-year-long evolution, approximately. As for the intraoral physical examination, a reddish nodular lesion was found in the lower lip, on the right side, close to the labial commissure, measuring approximately $1.0 \mathrm{~cm}$ in diameter, consisting of a sessile base, smooth surface, well-defined limits, purplish coloring, soft consistency, and no painful symptomatology on palpation. After the diascopy and clinical diagnosis of vascular injury, we opted for the photocoalulation with high-power diode laser. Results: Total regression of the lesion was seen after a single application with no trans- and post-operative complications, no bleeding during surgery, leading to better surgical conditions and a minimally invasive procedure. The patient is on proservation, with no recurrence of the lesion. Conclusion: Photocoagulation can be considered a safe alternative for the treatment of vascular lesions.

Descriptors: Vascular System Injuries; Light Coagulation; Lasers; Mouth Mucosa.

\section{Resumen}

Introducción: Las alteraciones vasculares son anomalias del desarrollo de los vasos sanguíneos que propician acumulación de sangre y son de etiologia desconocida. Entre ellas estan, las malformaciones arteriovenosas, hemangiomas, linfangiomas y varicosidades. Aunque no son raras, las lesiones vasculares de los lábios pueden ser antiestéticas y su diagnóstico y tratamiento son complejos. Objetivo: Describir un estudio de caso aplicando láser de diodo de alta potencia para la fotocoagulación de una lesión vascular, abordando sus ventajas y limitaciones en comparación con el tratamiento quirúrgico. Material y Método: Paciente de 53 años, leucoderma, quién relató la aparición de una burbuja de sangre en el labio con una evolución de aproximadamente ocho años. Al examen físico intraoral, se observó un nódulo en el bermellón del labio inferior derecho, cerca de la comisura labial, de aproximadamente $1.0 \mathrm{~cm}$ de diámetro, con base sésil, superficie lisa, límites bien definidos, coloración violeta, consistencia suave y sin síntomas dolorosos a la palpación. Después de la diascopia y el diagnóstico clínico de la lesión, se eligió la fotocoagulación con láser de diodo de alta potencia como tratamiento. Hubo regresión total de la lesión después de una sola aplicación, sin complicaciones trans y post-operatorias y sin sangrado durante la cirugía, lo que proporcionó una mejor visión del campo operatorio y fué mínimamente invasivo. La paciente está en observación periódica y sin recidiva. Conclusión: La fotocoagulación puede considerarse como una alternativa segura y eficiente para el tratamiento de lesiones vasculares.

Descriptores: Lesiones del Sistema Vascular; Fotocoagulación; Rayos Láser; Mucosa Bucal.

\section{INTRODUÇÃO}

As alterações vasculares (AV) são anomalias de desenvolvimento dos vasos que se caracterizam pelo acúmulo de sangue e são de etiologia desconhecida, podendo ser encontradas em qualquer parte do corpo, no entanto apresentam alta prevalência na região de cabeça e pescoço ${ }^{1}$.

Segundo a Sociedade Internacional para o
Estudo de Anomalias Vasculares (ISSVA), as anomalias vasculares podem ser didaticamente classificadas em malformações simples, malformações combinadas, malformações de vasos maiores e malformações associadas a outras anomalias $^{2,3}$

As AV apresentam altos índices de 
incidência na cavidade oral, sendo a superfície ventral da língua, lábios e comissura labial as localizações mais prevalentes. No entanto pode acometer, em menores frequências, as regiões de mucosa jugal, gengivas e palato ${ }^{4,5}$.

$\mathrm{O}$ hemangioma, proliferação benigna de células endoteliais, é a neoplasia mais comum da infância, ocorrendo em 10 a $12 \%$ das crianças com um ano de idade e atingindo até $30 \%$ daquelas com peso muito baixo ao nascimento (menos de $1.000 \mathrm{~g})^{6}$. Frequentemente não está presente no nascimento e desenvolve-se em três fases: proliferativa, regressiva e involuída. Pode apresentar coloração que varia do vermelho ao roxo a depender da localização e da profundidade da invasão tecidual, bem como o grau de congestão vascular da área afetada; além disso, podem apresentar-se planos ou elevados; com superfície lisa ou nodular; bordas delimitadas; séssil ou pedunculada e de consistência amolecidosà palpação. Podem ser pulsáteis, com uma temperatura mais elevada que os tecidos adjacentes ${ }^{7}$. As malformações vasculares ocorrem igualmente em ambos os sexos, apresentando-se como lesões únicas ou múltiplas e têm prevalência na região de cabeça e pescoço. Quando localizados na boca, lábios, língua ou mucosas, pode levar a alterações estéticas, dor, limitações funcionais, dificuldade na fonação e obstrução das vias aéreas superiores ${ }^{8}$. Chang et al. ${ }^{9}$ relataram que as malformações vasculares labiais devem receber atenção especial, pois podem alterar a anatomia do lábio e ter uma maior probabilidade de ulcerar e/ou sangrar, propiciando maior formação de cicatrizes e deformidades, o que compromete a estética facial do indivíduo.

A diascopia ou vitropressão constitui um importante meio auxiliar no estabelecimento do diagnóstico diferencial das AV e demais lesões que acometem o sistema estomatognático. Manobra em que a compressão exercida por uma lâmina de vidro contra a lesão, faz com que esta adquira uma coloração pálida e diminua de tamanho devido ao esvaziamento vascular ${ }^{10}$. Isso ocorre porque a pressão exercida pelo vidro na lesão comprime os vasos sanguíneos, fazendo-os extravasarem sangue para os vasos periféricos. Após a remoção da pressão, o sangue retorna para o interior das mesmas e recupera a sua aparência original podendo-se assim, estabelecer o diagnóstico clínico sugestivo, eliminando hipóteses de quaisquer lesões pigmentadas ${ }^{11}$.

As opções de tratamento para as AV incluem excisão cirúrgica ${ }^{12-14}$, terapia medicamentosa com o uso de corticosteroides sistêmicos ${ }^{14,15}$, escleroterapia $^{16}$, terapia a laser ${ }^{14,17}$, uso de interferon alfa $^{14}$ e também a associação dessas terapias. A escolha do método mais adequado deve considerar as suas vantagens, o efeito colateral encontrado, o perfil do paciente ${ }^{8,16,18}$, assim como o tamanho, localização e a condição clínica da lesão ${ }^{19,20}$.

Entre os métodos não cirúrgicos, há algumas evidências apontando para um sucesso clínico significativo após o tratamento com laser de alta potência (LAP) ${ }^{3}$. Em procedimentos realizados nos tecidos moles, o laser cirúrgico é considerado uma alternativa viável e vantajosa, quando comparado ao bisturi, devido à ausência de sangramento, menor formação de edema e um pós-operatório menos doloroso. Essa forma terapêutica possibilita, portanto, a realização de cirurgias de modo menos invasivo e agressivo $^{21}$. Os lasers mais utilizados e eficazes para esses tipos de procedimentos são $\mathrm{CO} 2, \mathrm{Nd}$ : YAG e $\operatorname{diodo}^{22}$.

Diante do exposto, o objetivo deste trabalho foi relatar e discutir caso clínico de uma lesão vascular em lábio tratado com fotocoagulação a laser de diodo. O procedimento foi realizado na Clínica Escola do Departamento de Odontologia da Universidade Estadual da Paraíba, Campina Grande - PB, Brasil. Paciente concordou com a divulgação de seu caso para fins científicos mediante assinatura de termo de consentimento livre e esclarecido antes e após a realização do procedimento.

\section{CASO CLÍNICO}

Paciente de 53 anos de idade, branca, sexo feminino, procurou o serviço da Clínica-Escola do Departamento de Odontologia da Universidade Estadual da Paraíba (UEPB) queixando-se do aparecimento de "uma bolha de sangue no lábio" sem sintomatologia dolorosa, com evolução de aproximadamente oito anos. Durante a anmnese, a paciente não relatou alteração sistêmica. $O$ exame físico extraoral não revelou linfonodos cervicais palpáveis ou lesão cutânea. Ao exame físico intraoral, constatou-se uma lesão nodular, localizada no vermelhão do lábio inferior, lado direito, próxima à comissura labial, medindo aproximadamente $1,0 \mathrm{~cm}$ de diâmetro, base séssil, superfície lisa, limites bem definidos, de coloração violácea e consistência macia (Figura 1).

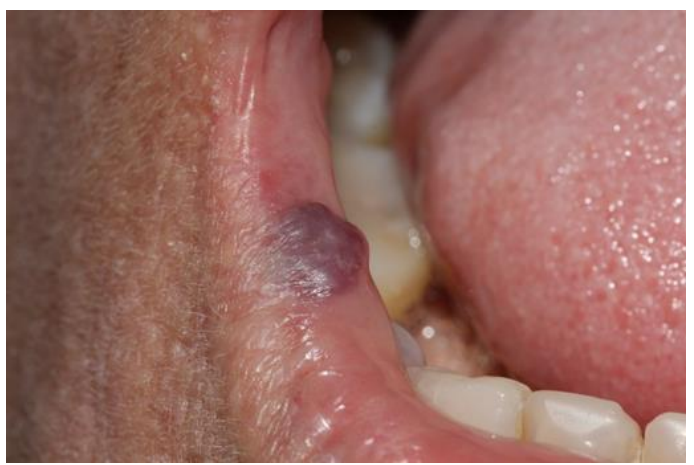

Figura 1: Aspecto clínico da lesão antes da fotocoagulação, demonstrando aspecto nodular de coloração violácea em região de lábio sugestiva de alteração de origem vascular.

Após a realização da diascopia, com o objetivo de fazer o diagnóstico diferencial com outras lesões 
clinicamente semelhantes, observou-se isquemia na região, obtendo-se a hipótese diagnóstica de $\mathrm{AV}$. Desta forma, optou pela fotocoagulação com laser diodo de alta potência (TheraLaseSurgery® - DMC Equipamentos, São Carlos, Brasil), no comprimento de onda Infravermelho $(808 \mathrm{~nm})$, potência $1,5 \mathrm{~W}$. A técnica utilizada consistiu na aplicação do laser de modo contínuo, pontualmente, com incidência no sentido perpendicular à lesão, estando a fibra óptica à 2,0 mm de distância da mesma, sob constante aspiração. $\mathrm{O}$ procedimento foi realizado em ambulatório sob anestesia local, realizada pela aplicação de $1 / 3$ de tubete do anestésico (Cloridrato de Mepivacaína a 2\%, com vasoconstrictorMEPADRE® Nova DFL), em sessão única. Não foi prescrito nenhum medicamento pós-operatório. No pós-operatório imediato, observou-se que a ação do laser ocasionou isquemia na região submetida à aplicação, além de ulceração como consequência do contato com a superfície da mucosa (Figura 2). Após 21 dias de tratamento, observou-se discreta área esbranquiçada no local da lesão (Figura 3). Paciente segue em proservação há dois anos, sem evidências de recidiva da lesão.

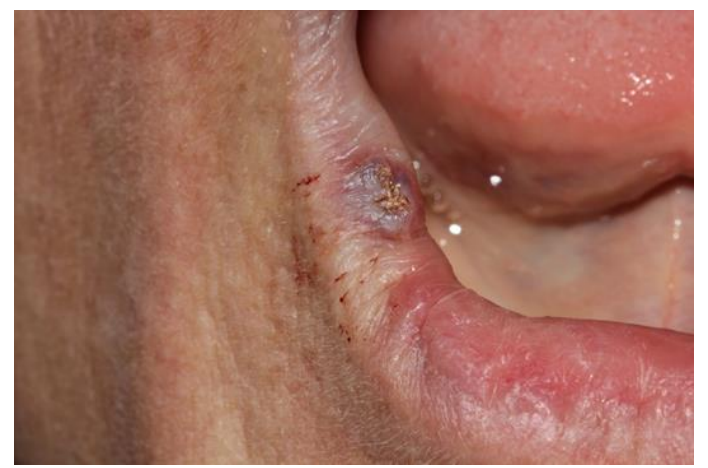

Figura 2: Aspecto clínico no pós-operatório imediato, isquemia e úlcera resultante da ação do laser e do seu contato com a superfície da mucosa, respectivamente.

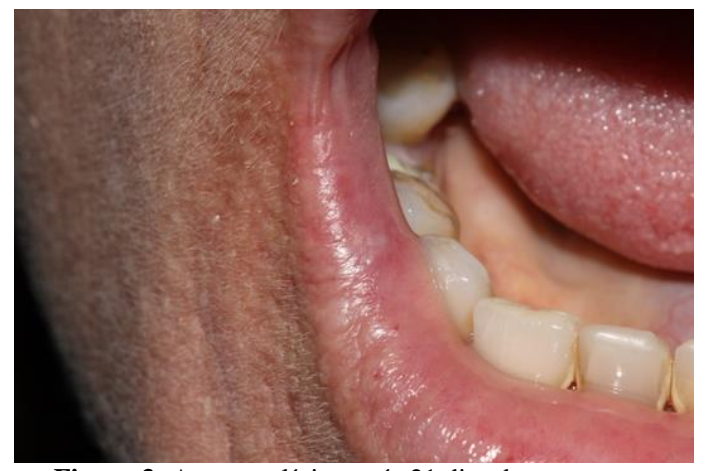

Figura 3: Aspecto clínico após 21 dias de tratamento.

\section{DISCUSSÃO}

O fato das lesões vasculares serem, na maioria dos casos, assintomáticas, faz com que a procura por tratamento por parte dos indivíduos acometidos por este tipo de alteração esteja fortemente relacionada ao comprometimento estético facial que pode vir a ser ocasionado por ela, o que interfere na autoestima destes indivíduos e gera, consequentemente, limitações na vida dos mesmos ${ }^{23}$.
$\mathrm{O}$ presente relato de caso representa uma constatação do estudo de Saawarn et al. ${ }^{23}$ uma vez que, a apesar do pequeno tamanho da lesão, a paciente apenas procurou os serviços de tratamento devido à sua queixa estética.

No estudo em questão, relata-se o tratamento de uma lesão de origem vascular presente em comissura labial, medindo aproximadamente 1,0 $\mathrm{cm}$. As características de tamanho e localização anatômica apresentadas no caso clínico corroboram com as pesquisas desenvolvidas por Angelo et al. ${ }^{4}$, Abdyli et al. ${ }^{5}$ e Choi Be et al. ${ }^{6}$, os quais afirmam que apesar da possibilidade das AV acometerem qualquer parte do corpo, estas alterações apresentam alto índice de incidência na cavidade oral, sendo a superfície ventral da língua, lábios e comissura labial as localizações mais prevalentes. Além disso, afirmam também que essas alterações apresentam variações de tamanho, que podem ir de poucos milímetros a vários centímetros.

Ribas et al. ${ }^{10}$, Correa et al. ${ }^{24}$, Fonseca et al. $^{25}$, Costa et al. ${ }^{8}$, Bharti e Singh ${ }^{26}$, Gill et al. ${ }^{12}$ e Mandu et al. ${ }^{16}$ demonstram em seus estudos, que entre os aspectos clínicos mais comuns das AV presentes em região de tecido mole, estão as lesões em forma de pápula ou nódulo, de coloração avermelhada, consistência resiliente à palpação, com superfície moriforme ou lisa. O caso clínico exposto no presente relato reafirma estes estudos, levando em consideração a semelhança no aspecto e na consistência pela presença de uma lesão em formato de nódulo mole à palpação. No entanto, a lesão encontrada neste caso apresentou uma coloração violácea, característica menos frequente neste tipo de alteração.

Percebe-se, então, a grande variedade de apresentações clínicas das lesões de origem vascular. Diante disso, ressalta-se que, assim como foi preconizado durante a realização do presente caso clínico, é indispensável lançar mão de manobras complementares como a diascopia, para que se confirme a presença do componente vascular neste tipo de alteração e, consequentemente, possibilite o correto diagnóstico clinico. Além disso, destaca-se a importância de considerar as necessidades individuais de cada paciente para a seleção da terapia mais apropriada e eficiente.

A escolha da conduta terapêutica mais adequada para o tratamento de AV depende de parâmetros como tipo e localização da lesão, proximidade desta alteração com estruturas vitais, idade do paciente acometido, danos estéticos e/ou funcionais que podem ser ocasionados por estas lesões ${ }^{27}$.

Sabe-se que durante muitos anos a excisão cirúrgica foi considerada o tratamento de eleição para $\mathrm{AV}^{28}$, entretanto, esta técnica estava sendo frequentemente associada a episódios de desconforto 
e hemorragia no período pós-operatório, o que gerou a necessidade de se pesquisar métodos menos invasivos, que pudessem proporcionar além de um campo cirúrgico sem sangramento, também uma diminuição no risco de formação de cicatriz ${ }^{8}$.

Diante disso, diversas modalidades de tratamento foram desenvolvidas e uma série de terapias alternativas ao tratamento convencional tem sido relatada na literatura, incluindo a utilização de corticosteroides orais, crioterapia, agentes esclerosantes, embolização, radiação e laserterapia $^{27,29,30}$.

Nesse contexto a laserterapia tem demonstrado resultados promissores no tratamento das malformações vasculares presentes em regiões de mucosa e da pele, tendo em vista que esta técnica inibe o sangramento local, dispensa a necessidade de suturas e previne o desenvolvimento de processos infecciosos, tal como demonstrado nos estudos de Monteiro et al. ${ }^{31}$ e Pedron et al. ${ }^{32}$, e reforçado no presente relato de caso clínico, o qual confirmou que a fotogoagulação efeutada pelo laser levou à preservação das funções estomatognáticas e ocasiocou danos mínimos aos tecidos adjacentes.

O Laser Diodo (810-1064 nm) é bastante utilizado na Odontologia por causa do seu pequeno porte, baixo custo e facilidade de uso para pequenas cirurgias $^{37}$. Jasper et al. ${ }^{33}$, ao avaliarem a experiência clínica do uso do laser de diodo para o tratamento de malformações vasculares no campo oral, verificou uma redução do tamanho de lesões vasculares por meio de procedimento intersticial e uma maior regressão destas malformações proporcionada pela fotocoagulação.

Ortega-Concepción et al. $^{34}$, ao revisarem a aplicação do laser diodo em lesões de tecidos moles orais, concluíram que esta terapia é de fácil aplicação e baixo custo, possui coagulação adequada, dispensa realização de sutura, provoca menor inflamação e dor, consequentemente, permite melhor reparo e recuperação. Frigerio e Tan ${ }^{36}$, por sua vez, afirmaram que outra vantagem do uso do laser é a capacidade de realizar esses procedimentos sob anestesia local, ao explicar que a isquemia pode diminuir o efeito do laser de diodo nos tecidos alvo, tendo em vista que este tem afinidade pela hemoglobina presente no sangue.

Durante a realização do tratamento proposto no caso clínico relatado neste estudo, foi possível identificar diversos dos benefícios associados à laserterapia, levando em consideração que a utilização de uma pequena quantidade de anestésico (1/3 de tubete sem vasoconstrictor) se mostrou suficiente para a realização do procedimento de forma satisfatória, além disso, a realização de sutura foi dispensável e não houve intercorrências no período pós-operatório, corroborando com o que foi obervado por Asnaashari e Zadsirjan ${ }^{37}$, Frigerio e
$\operatorname{Tan}^{35}$ (2015), Jasper et al. ${ }^{33}$ e Ortega-Concepción et al. ${ }^{34}$. Devido ao pequeno tamanho da lesão vascular do presente caso, foi proposta apenas uma única sessão de fotocoagulação no plano de tratamento, assim como ressaltado por Kobayashi et al. ${ }^{19}$, que afirma que o número de sessões de fotocoagulação a laser é diretamente relacionado ao tamanho da lesão. Dessa forma, foi possível alcançar um processo de cura sem cicatrizes ou perfusões, nenhuma dor pósoperatória foi reportada pela paciente, ressalta-se também que a presença de cicatrizes foi inexistente, descartando a necessidade de uma nova intervenção, reforçando o que foi observado por outros pesquisadores como Angiero et al. ${ }^{36}$ e Jasper et al. ${ }^{33}$ ao também utilizarem o laser de diodo e alcançarem resultados semelhantes aos que foram encontrados neste caso clínico.

Observou-se que os pontos positivos proporcionados pela técnica executada neste estudo, especialmente, no período pós-operatório, no que se refere ao conforto e ao curto tempo de recuperação, gerou satisfação no individuo que foi submetido à aplicação do laser. Reforçando o estudo de Fekrazad, Kalhori e Chiniforush ${ }^{20}$, o qual sugere que esta técnica tem boa aceitação pelos pacientes.

As terapias para as AV são múltiplas e demandam ainda muitas discussões e estudos, sendo assim, não há um tratamento universalmente aceito, mas sim aquele indicado para cada caso ${ }^{38}$. Apesar da fotocoagulação a laser ser uma modalidade terapêutica que demonstra diversos efeitos positivos no tratamento de anomalias vasculares benignas, atenta-se que para alcançar esses benefícios é indispensável que o operador seja habilitado para conhecer e aplicar os corretos parâmetros do laser, atrelado ao uso de um protocolo coerente, o bom manejo do paciente e à uma conduta cabível para evitar possíveis complicações trans e pós-operatórias.

\section{CONSIDERAÇÕES FINAIS}

A aplicação do laser de diodo cirúrgico pode ser considerada uma opção terapêutica segura e vantajosa para o tratamento de lesões vasculares em relação ao método cirúrgico convencional

\section{REFERÊNCIAS}

1. Nair SC, Spencer NJ, Nayak KP, Balasubramaniam K. Surgical management of vascular lesions of the head and neck: a review of 115 cases. Int $\mathrm{J}$ Oral Maxillofac Surg. 2011;40(6):577-83.

2. Dasgupta R, Fishman SJ. ISSVA Classification. Semin. Pediatr.Surg. 2014;23(4):158-61.

3. Medeiros R, Silva IH, Carvalho AT, Leão J. C.Nd:YAG laser photocoagulationofbenign oral vascular lesions: a case series. Lasers in Medical Science. 2015;30(8):2215-20.

4. Abdyli RA, Abdyli Y, Perjuci F, Gashi A, Agani 
Z, Ahmedi J. Slerotherapy of intraoral superficial hemangioma. Case rep dent. 2016;2016:1-5.

5. Choi BE, Kim Y, Leem DH, Baek JA, Ko SO. Utility of sodium tetradecyl sulfate sclerotherapy from benign oral vascular lesion. Maxillofac plast reconstr surg. 2016;38(1):1-4.

6. Angelo AR, Moraes JJC, Da Rosa MRD. Incidência de hemangioma na região de cabeça e pescoço em pacientes com faixa etária entre 0 e 18: estudo de 10 anos. Rev Odontol Univ São Paulo. 2008;20(2):209-14.

7. Palma FR, Garcia JAC, Jung R, Garcia RN, Aranha FCS. Escleroterapia de hemangioma oral. Relato de caso. SALUSVITA. 2016;35(1):85-93.

8. Costa JRS, Torriani MA, Hosni ES, D'Avila OP, Figueiredo PJ. Sclerotherapy for Vascular Malformations in the oral and Maxillofacial Region: Treatment and Follow-Up of 66 Lesions. J Oral Maxillofac Surg. 2011;69(6):88-92.

9. Chang CS, Wong A, Rohde CH, Ascherman JA, $\mathrm{Wu}$ JK. Management of lip hemangiomas: Minimizing peri-oral scars. JPRAS. 2012; 65(2):163-68.

10. Ribas MO, Laranjeira J, Sousa MH. Hemangioma bucal: escleroterapia com oleato de etanolamina. revisão da literatura e apresentação de caso. Rev de Clín Pesq Odontol. 2004;1(2):31-6.

11. Silva WB, Ribeiro ALR, De Menezes SAF, Pinheiro JJV, Alves-Junior SM. Oral capillary hemangioma: A clinical protocol of diagnosis and treatment in adults. Oral Maxillofac Surg. 2013;18(4):431-37.

12. Gill JS, Gill S, Bhardwaj A, Grover HA Oral Hemangioma. Case Reports in Medicine. 2012; 2012:1-4.

13. Rezende KMP, Corrêa FNP, Corrêa JPNP, Bönecker M, Corrêa MSNP. Hemangioma: descrição de um caso clínico e sua importância no diagnóstico diferencial. Rev Assoc Paul Cir Dent. 2016;70(1):19-23.

14. Passas MA, Teixeira M. Hemangioma da infância. Nascer e Crescer. 2016;25(2):83-9.

15. Chen W, Zhang B, Li J, Yang Z, Wang Y, Huang $\mathrm{Z}$ et al. Liquid nitrogen cryotherapy of lip mucosa hemangiomas under inhalation general anesthesia with sevoflurane in early infancy. Ann Plast Surg. 2009;62(2):154-57.

16. Mandu ALC, Lira CRS, Barbosa LM, Silva VCR, Cardoso AJO. Escleroterapia de Hemangioma: relato de caso. Rev Cir Traumatol Buco-Maxilofac. 2013;13(1):71-6.

17. Silva AJDD, Dos Santos RV, Amato SJTA, Amato ACM. Malformação venosa associada à hiperelasticidade cutânea e atrofia do tecido subcutâneo. J Vasc Bras. 2016;15(1):66-9.

18. Gupta A, Verma A, Dhua A, Bhatnagar V. Vascular anomalies: a pediatric surgeon's perspective. Indian J Pediatr. 2017;84(8):612-17.
19. Kobayashi, K, Nakao K, Kishishita S, Tamaruya N, Monobe H, Saito K, Kihara A. Vascular malformations of the head and neck. Auris Nasus Larynx. 2013;40(1):89-92.

20. Fekrazad R, Am Kalhori K, Chiniforush N. Defocused irradiation mode of diode laser for conservative treatment of oral hemangioma. J Lasers Med Sci. 2013;4(3):147-50.

21. Tonioli IB, Tomo S, Boer NB, Simonato LE, De Lucia MBI. OR 12. Tratamento de hemangioma em lábio superior com agente esclerosante. Arch Health Invest. 2016;5(Spec Iss 3):77.

22. Tachmatzidis T, Dabarakis N. Technology of lasers and their applications in oral surgery: Literature review. Balk J Dent Med. 2016;20(3):131-37.

23. Corrêa PH, Nunes LC, Johann AC, Aguiar MC, Gomez RS, Mesquita RA. Prevalence of oral hemangioma, vascular malformation and varix in a Brazilian population. Braz Oral Res. 2007; 21(1):40-5.

24. Fonseca Junior LA, Cha SB, Cartumm J, Rehder JRCL. Eficácia terapêutica do interferon alfa em criança com hemangioma gigante craniofacial: relato de caso. Arq Bras Oftalmol. 2008; 71(3):423-26.

25. Bharti V, Singh J. Capillary hemangioma of palatal mucosa. J Indian Soc Periodontol. 2012; 16(3):475-78.

26. Saawarn N, Saawarn S, Ragavendra R, Kasetty S, Ekka RK, Singh V. Oral hemangioma management in children: a concise review. Inter Ped Dent Open Acc J. 2018;1(3):49-50.

27. Pereira ERD, Silva GLS, Sahium GMB, Pereira JPS, De Faria MM, Faria RBCC. Laser de Nd YAG1064 $\mathrm{ncm}$ no tratamento de hemangioma: relato de caso. Rev Educ Saúde. 2017;5(2):130-35.

28. Newadkar UR. Oral hemangioma or vascular malformation: different entities. J Indian Acad Oral Med Radiol 2015;27:497-99.

29. Biddappa L, Kanwar S, Lingaraju N, Kumaran S. A rare case of intraoral acquired hemangioma. Int J Health Sci Res. 2015;5(8):610-13.

30. Rao G, Tripthi PS, Srinivasan K. Haemostatic effect of $\mathrm{CO} 2$ laser over excision of an intraoral hemangioma. Int J Laser Dent. 2012;2(3):74-7.

31. Monteiro LS, Azevedo A, Cadilhe S, Sousa D, Faria C, Martins M. Laser treatment of vascular anomalies of oral cavity. Rev Port Stomatol Med Dent Maxillofac Surg. 2013;54(3):171-75.

32. Pedron IG, Ramalho KM, Moreira LA, Freitas PM. Association of two lasers in the treatment of traumatic fibroma: excision with Nd: YAP laser and photobiomodulation using InGaAIP: a case report. J Oral Laser Appl. 2009;9(1):49-53.

33. Jasper J, Camilotti RS, Pagnoncelli RM, Poli VD, da Silveira Gerzson A, Gavin Zakszeski AM. Treatment of lip hemangioma using forced 
dehydration with induced photocoagulation via diode laser: report of three cases. Oral Surg Oral Med Oral Pathol Oral Radiol. 2015;119(3):e89-94.

34. Ortega-Concepción D, Cano-Durán JA, PeñaCardelles J, Paredes-Rodríguez V, GonzálezSerrano J, López-Quiles J. The application of diode laser in the treatment of oral soft tissues lesions. a literature review. J Clin Exp Dent. 2017;9(7):925-28.

35. Frigerio A, Tan OT. Laser applications for benign oral lesions. Lasers Surg Med. 2015;47(8):643-50.

36. Angiero F, Benedicenti S, Benedicenti A, Arcieri $\mathrm{K}$, Bernè $\mathrm{E}$. Head and neck hemangiomas in pediatric patients treated with endolesional 980$\mathrm{nm}$ diode laser. Photomed Laser Surg. 2009;27(4):553-59.

37. Asnaashari M, Zadsirjan S. Application of laser in oral surgery. J Lasers Med Sci. 2014;5(3):97-107.

38. Silva TWS, Do Nascimento ACC, Ferreira Filho, JL. Diagnóstico e tratamento de hemangioma cavernoso intraoral - relato de caso. JOAC. 2018;4(1):1-6.

\section{CONFLITO DE INTERESSES}

Os autores declaram não haver conflitos de interesse.

\section{AUTOR PARA CORRESPONDÊNCIA}

\section{Daliana Queiroga de Castro Gomes}

Departamento de Odontologia

Universidade Estadual da Paraíba, UEPB

Rua Baraúnas, 351, Bairro Universitário

58429-500 Campina Grande- PB, Brasil

Email: dqcgomes@hotmail.com 